\title{
A new spacetime symmetry
}

\author{
Keith A. Fredericks \\ Restframe Labs, New York, NY, USA
}

(Dated: April 4, 2020)

\begin{abstract}
Based on detection of elliptic particle tracks, $\simeq 137^{2} n^{2}$ bigger than Bohr-Sommerfeld electron orbits, indicating the possible detection of superluminal electrons masquerading as magnetic monopoles, a new structure emerges leading to: (i) a new set of seven elementary lengths; (ii) replacement of the usual motion (Lorentz) transformations by scale transformations between $v^{2}<c^{2}$ and $v^{2}>c^{2}$ frames; (iii) equivalence of charge between $v^{2}<c^{2}$ and $v^{2}>c^{2}$ frames based on the Dirac-Schwinger quantization condition; (iv) a relativistic foundation for the Dirac-Schwinger quantization condition; (v) a possible cause of charge quantization; and (vi) the prospect of symmetry in Maxwell's equations. If the elliptic particle tracks are viewed as a magnification of electron orbits, the effect is suggestive of a spacetime distortion such as those predicted in general relativistic theories.
\end{abstract}

\section{INTRODUCTION}

Recent experiments [1] may provide insight into the development of a theory of faster-than-light particles. Detected quantized elliptic particle tracks with sizes $\simeq 137^{2} n^{2}$ bigger than Bohr-Sommerfeld electron orbits, associated with the Dirac quantization condition, lead to elementary lengths, which are mapped to extended relativity and result in a new type of superluminal transformation.

Historically, two main approaches to a theory of fasterthan-light particles, have been used [2], each focused on transformations to and from superluminal velocities:

1. Assuming that standard Lorentz transformations can be used for superluminal velocities [3, 4], $\gamma=(1-\beta)^{-1 / 2}$, where $\beta=v / c$ and $\beta>1$ and rest mass, proper length and proper time become imaginary, but this may not be important since it is not possible to measure these with $v^{2}<c^{2}$ observers $[3,4]$.

2. Assuming that equivalent $v^{2}<c^{2}$ and $v^{2}>c^{2}$ frames exist and that it is possible to perform standard Lorentz transformations between them, the Lorentz factor becomes $\gamma=(\beta-1)^{-1 / 2}$, where $\beta=v / c$ and $\beta>1$ [5-7]. Here, inertial frames, moving at superluminal relative velocities, partitioned at the speed of light, co-exist in our universe. Within each frame physicists observe the very same laws. This approach allows observers in $v^{2}>c^{2}$ frames, but, in four-dimensions, certain imaginary quantities enter the equations that cannot be measured [8, 9]. [10] [11]

We present a completely new approach based on the equivalence of frames, but in contrast to equivalent $v^{2}<c^{2}$ and $v^{2}>c^{2}$ frames, equivalent $\ell^{2}<d^{2}$ and $\ell^{2}>d^{2}$ frames are proposed. A theory of faster-thanlight particles may be constructed using inertial frames with lengths greater than a certain length, $d$ rather than inertial frames with speeds greater than $c$. This new approach preserves important properties of earlier theories but improves important features. Scale transformations rather than motion transformations connect observations between equivalent frames. Extended relativity is modified as follows ( $\alpha$ is the fine structure constant, $g$ is magnetic charge, $\beta=v / c, A=\ell / d$ and $d$ is a new elementary length described in the text):

\begin{tabular}{lcc} 
& ER & new \\
charge & $g=e c$ & $g=\frac{e c}{\alpha}$ a \\
coupling & $\alpha$ & $\alpha_{\mathrm{EM}}= \begin{cases}\alpha, & \text { if } A<1 \quad \text { b } \\
\alpha^{-1}, & \text { if } A>1\end{cases}$ \\
transform & $\gamma=\left(\beta^{2}-1\right)^{-1 / 2}$ & $\alpha^{2}=\left(A^{2}-1\right)^{-1 \quad \text { c }}$ \\
\hline
\end{tabular}

a See section VI and section VII C.

b See Equation 7 .

c See section V.

Problems associated with faster-than-light particles, magnetic monopoles, charge quantization and electricmagnetic symmetry can be analyzed using this new model.

TABLE I. Symmetric Spacetime - Velocity and length mapped to $v^{2}>c^{2}$ and $v^{2}<c^{2}$ frames. The vertical interval between all values is $\alpha$. Type III ) Tachyons with $k_{m} g^{2}$ and coupling constant $\alpha^{-1}$. Type II ) Luxons with velocity $c$ and length $d$. Type I ) Bradyons with $k_{e} e^{2}$ and coupling constant, $\alpha$.

\begin{tabular}{|c|c|c|c|c|c|c|}
\hline type & velocity & $(\mathrm{m} / \mathrm{s}$ & & & lengt & $\mathrm{h}(\mathrm{m})$ \\
\hline III. & $\begin{array}{l}7.72 \times 10^{14} \\
5.63 \times 10^{12} \\
4.11 \times 10^{10}\end{array}$ & $v_{0 m}$ & $\begin{array}{l}c \alpha^{-3} \\
c \alpha^{-2} \\
c \alpha^{-1}\end{array}$ & $\begin{array}{l}d \alpha^{-3} \\
d \alpha^{-2} \\
d \alpha^{-1}\end{array}$ & $\begin{array}{l}r_{0 m} \\
\chi_{c m} \\
a_{0 m}\end{array}$ & $\begin{array}{l}1.86 \times 10^{-2} \\
1.36 \times 10^{-4} \\
9.93 \times 10^{-7}\end{array}$ \\
\hline II. & $2.99 \times 10^{8}$ & $c$ & $c \alpha^{0}$ & $\overline{d \alpha^{0}}$ & $d$ & $7.25 \times 10^{-9}$ \\
\hline I. & $\begin{array}{l}2.19 \times 10^{6} \\
1.60 \times 10^{4} \\
1.17 \times 10^{2}\end{array}$ & $v_{0 e}$ & $\begin{array}{l}c \alpha^{1} \\
c \alpha^{2} \\
c \alpha^{3}\end{array}$ & $\begin{array}{l}d \alpha^{1} \\
d \alpha^{2} \\
d \alpha^{3}\end{array}$ & $\begin{array}{l}a_{0 e} \\
\lambda_{c e} \\
r_{0 e}\end{array}$ & $\begin{array}{l}5.29 \times 10^{-11} \\
3.86 \times 10^{-13} \\
2.82 \times 10^{-15}\end{array}$ \\
\hline
\end{tabular}




\section{EXPERIMENTS}

In 1979, a technique was found using photographic emulsions that resulted in unusual particle tracks that had not been observed according to the scientific literature. The technique consists of "supplementary" uniform photon exposures on photographic emulsions. In total darkness or with the appropriate safelight, a relatively uniform exposure of photons is created from white LEDs of about $1200 \mathrm{mcd}$ at a distance of $6 \mathrm{~cm}$ from the surface of a lith emulsion such as Arista Ortho Litho 3.0 for a time of about $250 \mathrm{~ms}$. Any additional imagewise exposures, during exposure to the ambient environment, as in a cosmic ray study, will be amplified using this technique. $[1,12,13]$.

In 2002, Russian researchers reported similar tracks during experiments with electric explosion of metal wires and foils in water [14] and low-energy discharges in water [15]. It is clear, by comparison, that the same tracks were recorded with both supplementary photon exposures and electric discharges in these and other experiments $[12$, 13].

Microscopic examination of these tracks reveals structure consistent with particles traveling through emulsions (see elliptic track structure in Fig. 4 of Ref. [1]). A $1 / r^{2}$ central force would be required to create elliptic particle tracks in any nuclear track detector. The elliptic tracks are excellent fits for ellipses [1]. Particle-like structure resulting from $1 / r^{2}$ central force strongly indicates that these detected tracks are caused by particles.

In 2014, experiments were undertaken to determine if these particles could be affected by electric or magnetic fields [1]. Although no repeatable particle curvature resulted from applied electric or magnetic fields, elliptic tracks were found, often in conjunction with decay events [1]. The elliptic tracks add to the known properties [12] of the detected particle tracks. The elliptic tracks often occur at a point of decay of a primary track [1] (see Figure 1 and Figure 4) and differ from the predominant detected tracks [12]. Since it was shown that curvature, due to applied electric or magnetic fields, has not been observed for the predominant tracks, it was conjectured [1] that the predominant tracks are neutral and possibly comprised of oppositely charged particles and that these neutral composite particles may decay or split apart into separate charged particles. The separate charged particles may then become captured into elliptic orbits.

The track formation mechanism is unknown and paradoxical. In earlier work [1], we pointed out that

(i) particle tracks in photographic emulsions with smooth curvature are not known to occur for any known particle, yet these are commonly observed for the tracks under consideration.

(ii) although there is no high energy source for the tracks, estimates of deposited energy is quite large (up to $\sim 250 \mathrm{GeV} / \mathrm{cm}$ or greater).

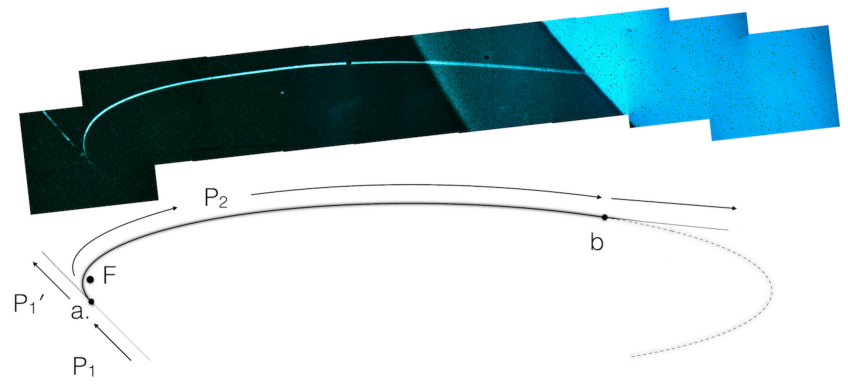

FIG. 1. Track $m e, n=7$. Top.) Semi-major axis size $a=$ $2391.6 \pm 16.1 \mu \mathrm{m}$. Ten tile photomosaic at 160x. Bottom.) Capture into and escape from an elliptic orbit. 1.) initial particle, $P_{1}$, trajectory. 2.) at point a. particle decays into $P_{1}^{\prime}$, continuing on initial trajectory and $P_{2}$, which is captured into an elliptic orbit. 3.) at point b. particle escapes from the elliptic orbit.

(iii) for Bohr-Sommerfeld elliptic tracks, we should see no energy loss from an elliptic orbit. By definition, these orbits have no dissipation and no energy loss. Furthermore, there is no known way to directly record an orbiting particle on a material detector, that is, where the particle would actually traverse the material.

Evidently our normal understanding of track formation does not apply in this case. Against this backdrop, we have created an idealized model of the elliptic tracks based on the measured track geometry.

Out of a population of 750 exposures, 22 tracks with elliptic curvature were selected. The semi-major axis size of these tracks were measured to be $\simeq 137^{2} n^{2}$ larger than Bohr-Sommerfeld electron orbit semi-major axis sizes. In addition, the measured elliptic particle tracks were shown to be quantized (see Figure 2.).

\section{SYMMETRIC WORLDS}

Dirac introduced the idea of electric charge quantization using the quantization condition [16] where $g$, magnetic charge is

$$
g=\frac{e c n}{2 \alpha}=n g_{D}, \quad n \in \mathbb{Z},
$$

where $\alpha=k_{e} e^{2} / \hbar c, k_{e} \equiv\left(4 \pi \varepsilon_{0}\right)^{-1}$ and $e$ is electric charge. This explanation of electric charge quantization due to magnetic monopoles (hereafter monopoles) continues to be the best yet found. With $n=2$, Eq. (1) becomes equivalent to the $n=1$ Schwinger quantization condition $[17]$

$$
g=\frac{e c}{\alpha}=2 g_{D}
$$

or, using shorthand, $g=2 g_{D}$.

From an analysis of the Dirac quantization condition, 


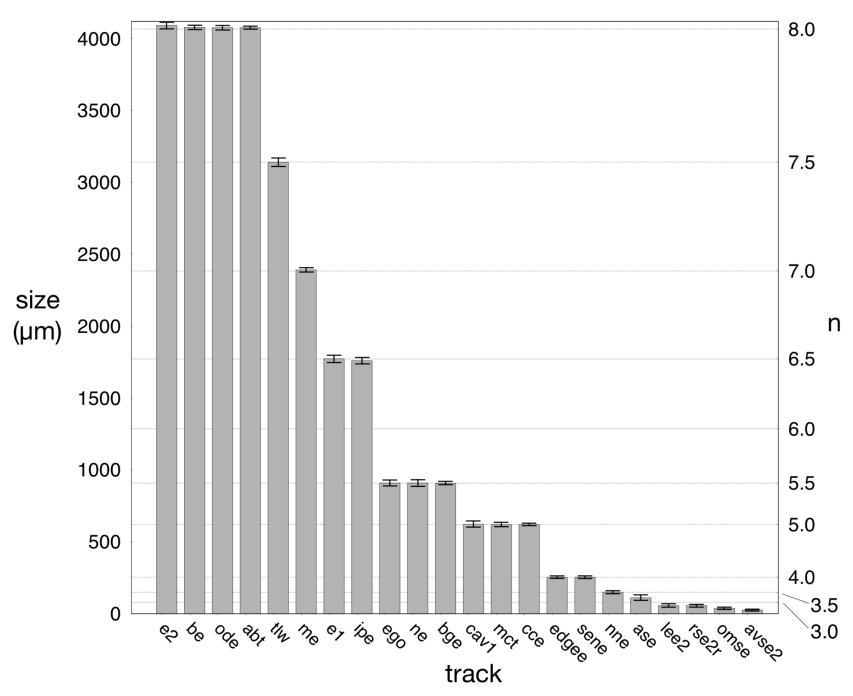

FIG. 2. Quantized ellipse semi-major axis sizes [1]. Ellipses between $n=8$ and $n=3.5$ are shown to be quantized as half integer values. Ellipses less than $n=3.5$ are quantized by quarter integer values. Error bars represent the combined standard uncertainty of the semi-major axis size.

using Eq. (1) with $n=1$, Datta [18] computed new elementary monopole lengths, $a_{0 m}, \lambda_{c m}$ and $r_{0 m}$, which are analogs of the Bohr radius, $a_{0 e}$, the reduced Compton wavelength, $\chi_{c e}$, (hereafter, Compton radius), and the classical electron radius, $r_{0 e}$, and showed that a hierarchy,

$$
a_{0 m}<\rtimes_{c m}<r_{0 m}
$$

exists as well as the usual hierarchy,

$$
a_{0 e}>\rtimes_{c e}>r_{0 e} .
$$

In this analysis, Datta

(i) used analogy with the electron for magnetic monopoles

(ii) reasoned that hierarchy (3) makes monopoles inherently relativistic ,

(iii) expressed concern that the size of the classical monopole radius, $r_{0 m}$, is so large that it would overlap with the anti-monopole with an orbital radius of $a_{0 m}$, and

(iv) found that a pair of north and south monopoles in an atom-like bound state would require superluminal ground state orbital speeds as in Eq. (6).

Datta used the simple analogy of the bound monopole and the bound electron. For example, since the ground state velocity of the bound electron is

$$
v_{0 e}=k_{e} \frac{e^{2}}{\hbar}=c \alpha,
$$

the ground state velocity of the bound monopole should

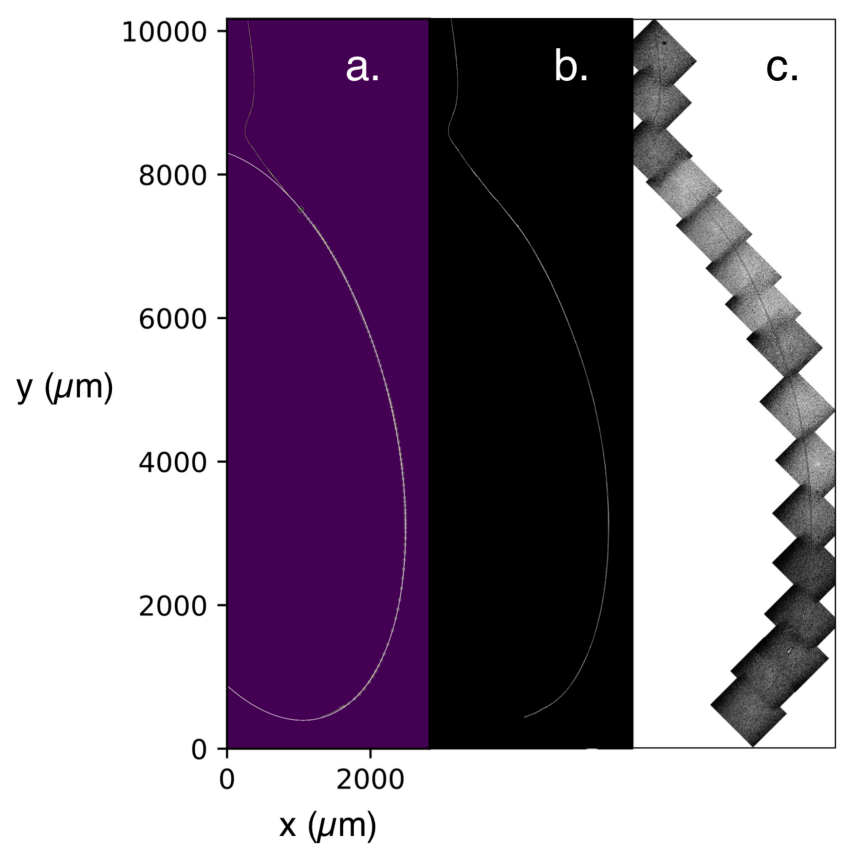

FIG. 3. Track be. The semi-major axis size of $a_{m}=4078.6 \pm$ $14.6 \mu \mathrm{m}$ is related to the semi-major axis size of an $n=8$ electron in hydrogen, $a_{e}$ by $a_{m} / 137^{2} n^{2} \simeq a_{e}$. a.) Leastsquares fit of $x, y$ track coordinates to ellipse overlayed on processed track image. b.) Track after image processing and background eradication. c.) Photo montage of track.

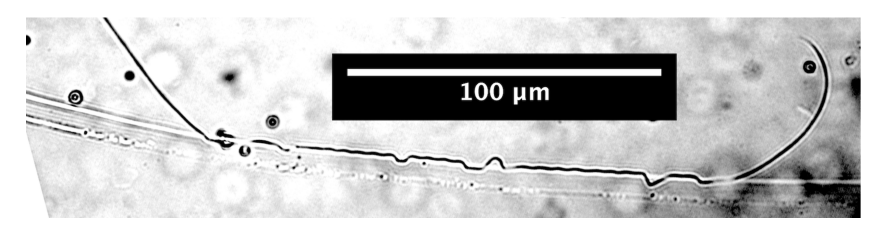

FIG. 4. Track rse2, $n=2.75$ showing elliptic track decay event. Photo at $400 x$.

be

$$
v_{0 m}=k_{m} \frac{g^{2}}{\hbar}=c / \alpha,
$$

where $\left(k_{m} \equiv \mu_{0} / 4 \pi\right)$. Due to points (iii) and (iv) (i.e. Eq. (6)), Datta rejected monopoles as unphysical, ending his investigation.

These specific points will be considered later (see section VA), but the mapping of hierarchy (3) to fasterthan-light monopoles in extended relativity warrants further investigation.

Continuing along these lines, first it is noted that using $g=2 g_{D}$, the coupling constant, $\alpha$, simply inverts with $\beta$. [19]

$$
\alpha_{\mathrm{EM}}=\left\{\begin{array}{ll}
\alpha, & \text { if } \beta<1 \\
\alpha^{-1}, & \text { if } \beta>1
\end{array} .\right.
$$

Here the notation is used that $\alpha_{e}=\alpha$ and $\alpha_{m}=1 / \alpha$ 
where $\alpha$ is the fine structure constant. Monopole mass can be found either by using the Compton radius of the monopole in terms of the Compton radius of the electron [19] or by using the analogy between the Bohr radius for the electon versus the Bohr radius for the monopole using the $1 / \alpha^{2}$ relation derived from experiments [1]. Here the second approach is used. Using $g=2 g_{D}$, the ratio of the monopole Bohr radius to the electron Bohr radius is

$$
\frac{a_{0 m}}{a_{0 e}}=\frac{1}{\alpha^{2}} .
$$

Using the formula for Bohr radius, $a_{0 e}=\hbar / m_{e} c \alpha$, the analogous $a_{0 m}=\hbar / m_{m} c \alpha_{m}$ and Eq. (8), monopole mass can be written as

$$
m_{m}=\frac{m_{e} \alpha^{3}}{\alpha_{m}},
$$

and since $\alpha_{m}=\alpha^{-1}$

$$
m_{m}=m_{e} \alpha^{4}=1.45 \times 10^{-3} \mathrm{eV} / \mathrm{c}^{2},
$$

which leads to the fine structure constant, $\alpha=$ $\left(m_{m} / m_{e}\right)^{1 / 4}$, as a mass ratio.

Using $g=2 g_{D}$, analogy with the electron, and monopole mass, Table I. can be populated with values. The electron and monopole Bohr radii are equally offset from the length, $d$, corresponding to the speed of light, $c$, such that the monopole Bohr radius, is scale symmetric with the electron Bohr radius. A similar symmetric relation exists between the ground state orbital velocities and $c$. Thus $c$ and $d$ form the axis of symmetry for both Bohr radii, the ground state orbital velocities and hierarchies (3) and (4) [19] (see Table I.).

Hierarchy (4) comprises a set of three elementary lengths incremented by the fine structure constant, $\alpha$. With the identification of $d=c t$ and hierarchy (3), The entire right side of Table I. represents a new set of seven elementary lengths, each incremented by $\alpha$.

\section{EXTENDED RELATIVITY}

Parker [5] notably forwarded the idea of extending relativity to superluminal frames. He was also the first to suggest that when special relativity is extended to superluminal velocities, charged tachyons should be detected as magnetic monopoles by subluminal observers.

Baldo, et.al [20], Recami and Mignani, [21] and Recami [22] developed Parker's concepts [23-27]. Since faster-than-light electric charge, in this theory, brings into Maxwell's equations exactly what magnetic monopoles would (see section VIII), subluminal monopoles were rejected and, using a symmetry argument, a magnetic charge of $g=-e c$ was initially proposed in accordance with charge invariance (although later Schwinger quantization was favored). As a result, the coupling constant, $\alpha$ took a singular value both above and below the speed of light. According to Recami, et.al slower-than-light monopoles do not exist.

The structure of extended relativity follows Bilaniuk, et.al's [3] original classification of particles. That is:

$$
\begin{aligned}
& \text { I. braydons }\left(v^{2}<c^{2}\right) \text {, } \\
& \text { II. luxons }(v=c) \text { and } \\
& \text { III. tachyons }\left(v^{2}>c^{2}\right) \text {. }
\end{aligned}
$$

The symmetric spacetime in Table I. maps one-to-one with the structure of extended relativity [5, 21, 22], i.e. symmetric $v^{2}>c^{2}$ and $v^{2}<c^{2}$ worlds partitioned at $c$, the speed of light, symmetric $\ell^{2}>d^{2}$ and $\ell^{2}<d^{2}$ worlds partitioned at $d$ (see section VII) or, more generally, type III and type I worlds partitioned by type II speed and length. Type I, type II, and type III will be used throughout in accordance with Table I.

The central feature of extended relativity is the superluminal Lorentz transformation (SLT). The SLT shows what a type III object in a type III frame looks like to an observer in a type I frame and so connects observations of type I observers with observations of type III observers.

The collinear bi-dimensional $(x, t)$ SLT works without problems, but, when extended to four dimensions, $(x, y, z, t)$, it leads to imaginary quantities $[8,9]$ for certain measurements of $v^{2}>c^{2}$ (type III) objects and problems of how to interpret these physically. It is possible to use a six-dimensional spacetime for the SLT [28], but it may be difficult to physically interpret two additional time dimensions.

The standard collinear bi-dimensional SLT in its usual form will not transform the detected ellipse semi-major axis size into a Bohr-Sommerfeld ellipse semi-major axis size. That is

$$
a_{m}^{(n)}(\gamma) \neq a_{e}^{(n)}, \quad \gamma=\left(\beta^{2}-1\right)^{-1 / 2} .
$$

Using Recami's multidimensional approach, the SLT inverts the quadratic form sign [29] of an object and a bound superluminal electron detected as a monopole ellipse takes the form

$$
0 \geq-\frac{x^{2}}{a_{m}^{(n) 2}}+\frac{y^{2}}{b_{m}^{(n) 2}} \geq-1,
$$

consisting of the area between an on-central-axis conic section,

$$
\frac{y^{2}}{b_{m}^{(n) 2}}=\frac{x^{2}}{a_{m}^{(n) 2}},
$$

and a hyperbola,

$$
\frac{y^{2}}{b_{m}^{(n) 2}}=\frac{x^{2}}{a_{m}^{(n) 2}}-1,
$$

radically distorted from the original ellipse with eccentricity, $\mathscr{E}^{\prime}>1$, as shown in Figure 5. [30] 


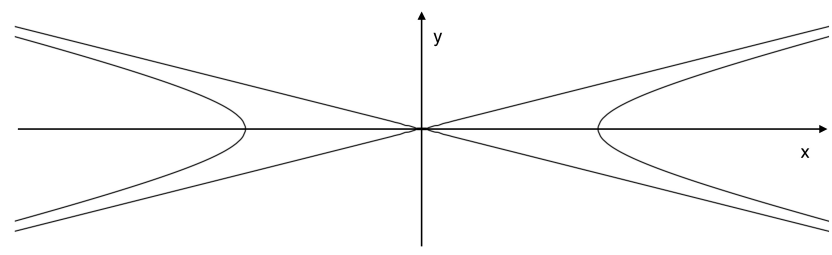

FIG. 5. Recami type SLT of a theoretical $n=8, l=2$ (example quantum numbers) monopole ellipse.

The elliptic particle tracks we observe do not share the same semi-major axis size as their (presumed) subluminal counterparts, that is, the Bohr-Sommerfeld hydrogen semi-major axis sizes. They are larger by $\alpha^{-2}$ but not distorted by stretching in one dimension.

The ratio of measured eccentricity, $\mathscr{E}_{\mu}$ to BohrSommerfeld eccentricity, $\mathscr{E}_{\tau}$

$$
\frac{\mathscr{E}_{\mu}}{\mathscr{E}_{\tau}}=\frac{\left(1-b^{2} / a^{2}\right)^{1 / 2}}{n^{-1}\left(n^{2}-\ell(\ell+1)\right)^{1 / 2}},
$$

as a ratio of averages over the 22 measured track values, $\left\langle\mathscr{E}_{\mu}\right\rangle /\left\langle\mathscr{E}_{\tau}\right\rangle=0.99$, so measured eccentricity corresponds well to Bohr-Sommerfeld electron orbit eccentricity. This implies that the (superluminal) transformation, for this restricted case of bound particles, should be a scale transformation.

Since measured eccentricity corresponds to pure BohrSommerfeld eccentricity, then, due to the $\alpha^{-2}$ size change, the transformation of ellipses in general needs to be an isotropic scale transformation instead of a translation along one axis.

\section{THE COULOMB FLIP}

The force difference between magnetism and electricity using $g=2 g_{D}, r_{e}=a_{0 e}$ and $r_{m}=a_{0 m}$ is the difference between Coulomb's law for magnetism and electricity

$$
\frac{F_{m}^{(n)}}{F_{e}^{(n)}}=\frac{k_{m} g^{2} / a_{0 m}^{(n) 2}}{k_{e} e^{2} / a_{0 e}^{(n) 2}}=n^{2} \alpha^{2} \simeq \frac{n^{2}}{137^{2}} .
$$

Since detected ellipses are the same shape but $137^{2} n^{2}$ bigger than Bohr-Sommerfeld electron orbits, the most direct explanation would be that these are BohrSommerfeld (type III) monopole orbits and the required transformation is identical to the force ratio, from Eq. (16) between magnetic and electric force strengths, which is a scale ratio, dilation $\left(\alpha^{-2}\right)$ or contraction $\left(\alpha^{2}\right)$, which can be called the Coulomb flip.

The magnetic and electric Bohr-Sommerfeld Kepler ellipses are completely analogous and scale symmetric with respect to the speed of light, $c$ and the length, $d$. Using $g=2 g_{D}$, the Kepler period for an electron in hydrogen and the "monopole" in a magnetic hydrogen analog,

$$
t_{m}^{(n)}=\frac{2 \pi a_{0 m} n^{3}}{v_{0 m}}=\frac{2 \pi a_{0 e} n^{3}}{v_{0 e}}=t_{e}^{(n)},
$$

are equal.

The one-dimensional basis Coulomb flip contractions from type III to type I are

$$
\begin{array}{rcc}
\text { coupling constant } & \alpha_{\mathrm{EM}}: & \alpha_{m} \quad\left(\alpha^{2}\right)=\alpha_{e} \\
\text { Bohr radius } & a_{0}: & a_{0 m} \quad\left(\alpha^{2}\right)=a_{0 e} \\
\text { ground state } v & v_{0}: & \alpha_{m} c\left(\alpha^{2}\right)=\alpha_{e} c \\
\text { charge } & q_{0}^{2}: & k_{m} g^{2}\left(\alpha^{2}\right)=k_{e} e^{2}
\end{array}
$$

Combining the basis transformations, Eqs. (18b), (18c), and (18d) and assuming $\hbar$ is Coulomb flip invariant, transformations between type III and type I frames for time, momentum, binding energy and mass are

$$
\begin{aligned}
& t_{m}=\frac{2 \pi a_{0 m}}{\alpha_{m} c} \underset{\left(\alpha^{2}\right)}{\stackrel{\left(\alpha^{2}\right)}{\longrightarrow}} \frac{2 \pi a_{0 e}}{\alpha_{e} c}=t_{e} \\
& p_{m}=\frac{\hbar}{a_{0 m}} \underset{\left(\alpha^{2}\right)}{\stackrel{(1)}{\longrightarrow}} \frac{\hbar}{a_{0 e}}=p_{e} \\
& E_{m}=-\frac{k_{m} g^{2}}{2 a_{0 m}} \underset{\left(\alpha^{2}\right)}{\stackrel{\left(\alpha^{2}\right)}{\longrightarrow}}-\frac{k_{e} e^{2}}{2 a_{0 e}}=E_{e} \\
& U_{m}=\frac{k_{m} g^{2}}{2 a_{0 m}} \underset{\left(\alpha^{2}\right)}{\stackrel{\left(\alpha^{2}\right)}{2}} \frac{k_{e} e^{2}}{2 a_{0 e}}=U_{e} \\
& m_{m}=\frac{\hbar}{a_{0 m} \alpha_{m} c} \underset{\left(\alpha^{2} \alpha^{2}\right)}{\stackrel{(1)}{\longrightarrow}} \frac{\hbar}{a_{0 e} \alpha_{e} c}=m_{e}
\end{aligned}
$$

and the monopole and electron properties can be transformed into one another using Coulomb flips with $g=$ $2 g_{D}$. Time, in Eq. (19a) and energy in Eq. (19c) are invariant (in the ground state).

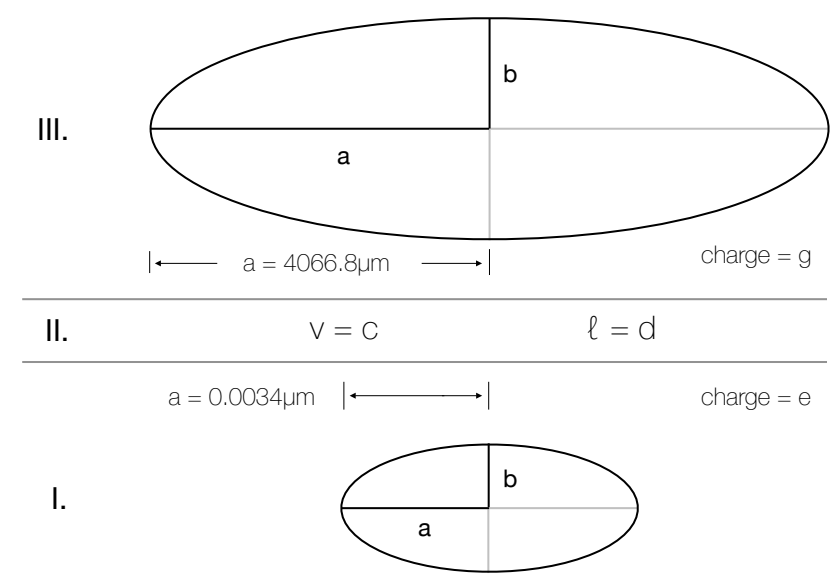

FIG. 6. How a type I observer sees a type I electron orbit and an $n=8, l=2$ type III "monopole" orbit (using example quantum numbers). The type III orbit is transformed to the type I orbit using the 2-dimensional Coulomb flip Eq. (20) (Not to scale). 
The 2-dimensional Coulomb flip scales (contracts) the parametric equation of the monopole ellipse (upper ellipse in Figure 6), by the factor $\alpha^{2} / n^{2}$,

$$
\left[\begin{array}{l}
x \\
y
\end{array}\right]=\frac{\alpha^{2}}{n^{2}}\left[\begin{array}{l}
a_{m}^{(n)} \cos \theta \\
b_{m}^{(n)} \sin \theta
\end{array}\right]=\left[\begin{array}{l}
a_{e}^{(n)} \cos \theta \\
b_{e}^{(n)} \sin \theta
\end{array}\right],
$$

where $0 \leq \theta \leq 2 \pi$, to the electron ellipse (lower ellipse in Figure 6).

The parametric equation to contract a sphere of $r=$ $a_{0 m}$ to $r=a_{0 e}$ using a scale factor of $\alpha^{2}$ is

$$
\left[\begin{array}{l}
x \\
y \\
z
\end{array}\right]=a_{0 m} \alpha^{2}\left[\begin{array}{ll}
\sin \theta & \cos \varphi \\
\sin \theta & \sin \varphi \\
\cos \theta &
\end{array}\right]=a_{0 e}\left[\begin{array}{ll}
\sin \theta & \cos \varphi \\
\sin \theta & \sin \varphi \\
\cos \theta &
\end{array}\right],
$$

where $0 \leq \theta \leq 2 \pi$ and $0 \leq \varphi \leq \pi$.

\section{A. Consequences}

Returning to hierarchies (3) and (4), type III observers will see what we refer to as hierarchy (3) as their normal electric hierarchy (4). In their home frame, type I observers and particles will recognize the type III electric charge as a magnetic charge. The mirror hierarchies map one-to-one with extended relativity, except that we are dealing with lengths (scale transformations) instead of velocities (Lorentz transformations).

Datta's points (ii) and (iii) above are nullified if hierarchy (3) is a type I to type III transformation of hierarchy (4) (that is, hierarchy (3) would just be the normal hierarchy (4) to type III observers). Datta's point (iv) is therefore relevant and could indicate the unrealized discovery of type III lengths and symmetric spacetime.

\section{TRANSFORMATION OF CHARGE}

Even though a type III particle's observed (by a type I observer) charge state is magnetic, it should possess exactly the same amount of charge in its own rest frame where the charge state is electric. This is to say that type III electric charge is observed by normal type I observers to be magnetic charge and that type I magnetic charge does not exist [23, 24].

As noted earlier, equivalence of charge along with considerations of special relativity motivated Recami, et.al. initially to propose $g=-e c$ and deviate from the Dirac-Schwinger quantization condition (although later Schwinger quantization was favored). In light of $g=2 g_{D}$ it is possible to reconsider this issue.

The basis Coulomb flip for charge in Eq. (18d) shows that, after transformation, the magnetic charge is equivalent to the electric charge in a type III frame observed from a type I frame.

Solving for $g$ in (18d) with $k_{m} / k_{e}=1 / c^{2}$ yields $g=e c / \alpha$, which is the Schwinger quantization condi- tion. So the Coulomb flip for charge is just the Schwinger quantization condition.

Consider a spherical condenser of size $a_{0 e}$. The electrostatic energy to charge the condenser to a total charge of $e$ is [31, (Eq. 8.11)]

$$
U_{e}=k_{e} \frac{e^{2}}{2 a_{0 e}},
$$

and the magnetostatic analog of Eq. (22) is

$$
U_{m}=k_{m} \frac{g^{2}}{2 a_{0 m}} .
$$

$U_{m}=U_{e}$ and from Eq. (19d),

$$
\frac{k_{m} g^{2}}{2 a_{0 m}}\left(\frac{\alpha^{2}}{\alpha^{2}}\right)=\frac{k_{e} e^{2}}{2 a_{0 e}},
$$

is the Coulomb flip between $U_{m}$ and $U_{e}$. All parameters of the magnetic condenser transform into the parameters of the electric condenser and vice versa. The amount of charge per capacity is equivalent.

This is a second application of the Coulomb flip, used for static spheres instead of bound states showing that the two spheres are equivalent and charge is invariant between subluminal and superluminal frames.

Transforming a spherical capacitor from a type III to a type I frame (or vice-versa) shows that magnetic charge is just electric charge in a type III frame as viewed from a type I frame. The equation for the transformation of the potential energy of the spherical capacitor is the Schwinger quantization condition $g=e c / \alpha$. This brings extended relativity into agreement with Dirac/Schwinger monopoles, but as Recami found, these monopoles are actually superluminal electrons and slower-than-light monopoles do not exist.

\section{RELATIVISTIC TRANSFORMATIONS}

In Table I. on the length side, the Bohr, Compton and classical electron radii clearly represent the radii of spheres, i.e. the basis for a three-dimensional or spatial scale. This stands in contrast to the one-dimensional velocity scale on the left side of the table upon which special relativity is based. Hierarchies (3) and (4) are equally separated from a length $d$ by $\alpha$. In Table I, the length $d$ corresponds to $c$. Many computations can use $d$ analogously to $c$. There is a direct correspondence between the transformation of velocities and the transformation of lengths between type III and type I frames as in

$$
\begin{aligned}
& v_{0 m}=c^{2} / v_{0 e}, \\
& a_{0 m}=d^{2} / a_{0 e} .
\end{aligned}
$$

The length $d$ is totally analogous with $c$. 


\section{A. Length transformations}

With the new elementary length $d$, it becomes possible to represent relativistic transformations equivalently using either length or velocity. Where $v$ is velocity on the left side of Table I. and $\ell$ is length on the right side of Table I., $v / c=\ell / d$ and so we can write

$$
\beta=A
$$

where $\beta=v / c$ and $A=\ell / d$. In addition, since the superluminal Lorentz factor is

$$
\gamma=\left(\beta^{2}-1\right)^{-1 / 2}, \quad(\beta>1),
$$

we can write the equivalent

$$
\alpha=\left(A^{2}-1\right)^{-1 / 2}, \quad(A>1) .
$$

Since $A=\beta$, the length-based forms can be substituted for the velocity-based forms in relativistic computations.

Because the lengths are three-dimensional spatial radii as opposed to a one-dimensional velocity vector, this affords a convenient way to differentiate between motionbased velocity transformations and the length-based scale transformations. Using this notation it is possible to show the $\alpha^{2}$ scale transformation (the Coulomb flip) as a relativistic transformation

$$
\alpha^{2}=\left(A^{2}-1\right)^{-1} .
$$

Eq. (29) can be seen as the relativistic factor between type I and type III worlds, replacing Eq. (27) of the SLT. That is, intra-frame transformations should use standard Lorentz transformations, i.e. $\gamma=\left(1-\beta^{2}\right)^{-1 / 2}$, and interframe transformations could use Coulomb flips.

\section{B. Relativistic expressions}

The relation of the two symmetric Compton radii, from Table I., converted to masses using $\chi_{m}^{-1}(\hbar c / e)$ and $\chi_{e}^{-1}(\hbar c / e)$, are at once seen as the electron and "monopole" masses and, in terms of relativity, if the electron mass $m_{e}=m_{0}$, i.e. the rest mass in our type I frame, then the "monopole" mass, $m_{m}=m^{\prime}$, is the "scale relativistic mass" as shown in Eq. (19e). The relativistic expression becomes

$$
m_{m}=\frac{m_{e}}{\left(A^{2}-1\right)^{2}}
$$

The same thing can be done for each of the Eqs. (18) and (19), substituting $\left(A^{2}-1\right)^{-1}$ for $\alpha^{2}$.

\section{The quantization condition}

In section VI it was shown that the Schwinger quantization condition constitutes the transformation of charge between type I and type III frames. The Schwinger quantization condition $g=e c / \alpha$ is also a Coulomb flip and can be represented as

$$
g=\frac{e c}{\left(A^{2}-1\right)^{1 / 2}}
$$

adding a relativistic interpretation to this quantum relation.

\section{MAXWELL'S EQUATIONS}

As has been mentioned previously [23], it is possible to recover symmetry in Maxwell's equations since type III particles with electric charge constitute magnetic charge in the equations. In extended relativity, Maxwell's equations for type I and type III particles, take a form

$$
\begin{aligned}
& \nabla \cdot D=\varrho_{e}(\mathrm{I}) \\
& \nabla \cdot B=-\varrho_{m}(\mathrm{III}) \\
& \nabla \times E=j_{m}(\mathrm{III})-\frac{\partial B}{\partial t} \\
& \nabla \times H=j_{e}(\mathrm{I})+\frac{\partial D}{\partial t}
\end{aligned}
$$

where $\mathrm{I}=$ type I frame and III = type III frame.

\section{THE $\alpha^{-2}$ X LENS}

If a type I observer peers into a type III frame, detects type III electrons and then transforms the detection using a Coulomb flip, this is equivalent to an $\alpha^{-2} \mathrm{x}$ magnification since type III electrons are by definition the same as type I electrons. This would mean that observing type III objects could provide a portal of magnification, analogous to gravitational lensing, into our own world such that $e . g$. electron orbits could be examined at $\alpha^{-2} \mathrm{x}\left(\sim 1.88 \times 10^{4} \mathrm{x}\right)$ magnification. This lens provides a way to visualize equivalent frames at different scales and their relationship.

\section{DISCUSSION}

Due to the sizes of detected elliptic particle tracks, the particles that created these tracks were found to have properties consistent with faster-than-light particles in an extended relativity theory. But the eccentricity of the detected ellipses, compared with Bohr-Sommerfeld ellipses, required the transformation between $v^{2}<c^{2}$ and $v^{2}>c^{2}$ frames to be an isotropic scale transformation rather than the SLT commonly used in extended relativity. 
A symmetric world is defined in Table I including both length and velocity-based values above and below a border delineated by $c$ and $d$ and generalizes these three regions into type I, type II, and type III frames.

To get between type I and type III frames, extended relativity models use Lorentz velocity transformations, represented by the left (velocity) side of Table I. Based on the right (length) side of this diagram, an alternative is considered to use scale transformations called Coulomb flips to transform between type I bound electron and type III bound "monopole" states. A number of Coulomb flips are shown in Eqs. (18) (19), (20) and (21).

This correspondence between the velocity-based and length-based transformations and the subsequent use of scale transformations brings into question the physical interpretation of the transformations. If the radius-based scale transformation is chosen, frames in extended relativity can be separated by scale rather than superluminal velocities and yet support superluminal relative velocities. The structure of this dual space is not clear, but it appears to have an analogy with the dual de Broglie wave.

If electrons from a type III frame were detected as magnetic charges in a type I frame, then Dirac's concept for the origin of charge quantization, defined as the interaction between magnetic charges and electrons should automatically become evidence for electromagnetic interaction between type I and type III worlds. A further implication of detecting electrons from a type III frame is to resolve the asymmetry between electricity and magnetism, thereby bringing symmetry to Maxwell's equations.

Charge in a type III frame can be transformed to charge in a type I. frame by means of a Coulomb flip. In addition, the transformation of charge from a type III to a type I frame can be represented as a relativistic transformation based on the Schwinger quantization condition. So the foundation for magnetic monopoles can be shown to be electric charge in a type III frame, which is connected with electric charge in a type I frame by a relativistic transformation. Recami and Mignani had shown this earlier by SLTs of $\boldsymbol{E}$ and $\boldsymbol{H}$ fields (see e.g. [21, 22])

In four-dimensional extended relativity, imaginary quantities $[8,9,32]$ occur in measurements between type I and type III frames due to the superluminal relative velocity. In the four-dimensional scale relativistic model, measurements occur between type I and type III frames at relative scales and are therefore limited to real quantities, so no imaginary quantities are possible. The scale relativistic model trivially solves the imaginary quantities problem.

\section{CONCLUSION}

Experimental results are discussed where quantized elliptic particle tracks $137^{2} n^{2}$ larger than Bohr-Sommerfeld electron orbits were detected [1]. Analysis indicated consistency between these particles and the properties expected of tachyon monopoles in extended relativity theories.

This led to the reexamination of a key discovery by Datta of superluminality resulting from the Dirac quantization condition, originally excluded [18], but now recovered [19]. This, in turn led to a symmetric spacetime structure in Table I forming a basis for theories of extended relativity.

An analysis of the experimental results using the symmetric spacetime structure leads to a new alternative isotropic scale transformation between type I and type III frames called the Coulomb flip.

The results presented here lead to possible solutions for problems of magnetic monopoles, charge quantization, equivalence of charge between type I and type III frames, imaginary quantities in four-dimensional SLTs and electric-magnetic symmetry in Maxwell's equations.

Since transformations of observations of type III particles constitutes the observation of a scale change, it is possible that simply observing type III particles is analogous to a gravitational lens.

Further experiments are required to study the elliptic tracks in greater detail using thicker $(10-100 \mu m)$ nuclear track emulsions with higher $(>80 \%) \mathrm{AgBr} /$ gelatin ratios.

\section{ACKNOWLEDGMENTS}

The author gratefully acknowledges stimulating discussions and review of Erasmo Recami without whose extensive body of work this would not have been possible.
[1] K. A. Fredericks, "Elliptical tracks: Evidence for superluminal electrons?" (2019), preprints, 2019070181.

[2] H. C. Corben, Int, J. Theor. Phys. 15, 703 (1976).

[3] O. M. P. Bilaniuk, V. K. Deshpande, and E. C. G. Sudarshan, Am. J. Phys. 30, 718 (1962).

[4] G. Feinberg, Phys. Rev. 159, 1089 (1967).

[5] L. Parker, Phys. Rev. 188, 2287 (1969).

[6] E. Recami and R. Mignani, Lett. Nuovo Cim. 4, 144 (1972).
[7] R. Goldoni, Lett. Nuovo Cim. 5S2, 495 (1972), [Lett. Nuovo Cim.5,495(1972)].

[8] E. Recami and G. D. Maccarrone, Lett. Nuovo Cim. (1971-1985) 28, 151 (1980).

[9] H. C. Corben, Lett. Nuovo Cim. (1971-1985) 11, 533 (1974).

[10] Recami and coworkers made significant advances, such as the (experimentally confirmed) X-shaped form of superluminal objects, despite the imaginary quantities, using 
the property of the superluminal Lorentz transformation (SLT) to invert the quadratic form sign. (see section IV).

[11] We agree with Corben on superluminal worlds [2] and imaginary quantities [9] but disagree (see section VI) that, except for speed, superluminal electric charge would be equivalent to subluminal electric charge [9? ].

[12] K. A. Fredericks, Eng. Phys. 6, 15 (2013).

[13] K. A. Fredericks, J. Condens. Mat. Nucl. Sci. 15, 203 (2015).

[14] L. I. Urutskoev, V. I. Liksonov, and V. G. Tsinoev, Ann. Fond. L. de Broglie 27, 701 (2002), arXiv:physics/0101089.

[15] N. G. Ivoilov, Ann. Fond. L. de Broglie 31, 115 (2006).

[16] P. A. M. Dirac, Proc. Royal Soc. A 133, 60 (1931).

[17] J. Schwinger, Phys. Rev. 144, 1087 (1966).

[18] T. Datta, Lett. Nuovo Cim. 37, 51 (1983).

[19] K. A. Fredericks, Phys. Essays 30, 269 (2017).

[20] M. Baldo, G. Fonte, and E. Recami, Lett. Nuovo Cim. (1969-1970) 4, 241 (1970).

[21] E. Recami and R. Mignani, Riv. Nuovo Cim. 4, 209 (1974).

[22] E. Recami, Riv. Nuovo Cim. 9, 1 (1986).

[23] R. Mignani and E. Recami, Lett. Nuovo Cim. 9, 367 (1974).
[24] E. Recami and R. Mignani, Lett. Nuovo Cim. 9, 479 (1974).

[25] R. Mignani and E. Recami, Lett. Nuovo Cim. 11, 417 (1974).

[26] E. Recami and R. Mignani, Phys. Lett. B 62, 41 (1976).

[27] E. Recami and R. Mignani, in The Uncertainty Principle and Foundations Of Quantum Mechanics, edited by W. C. Price and S. S. Chissick (Wiley, London, 1977) pp. 321-324.

[28] E. A. B. Cole, Il Nuovo Cim. A (1965-1970) 40, 171 (1977).

[29] A. Barut, G. Maccarrone, and E. Recami, Nuovo Cim. A 71, 509 (1982).

[30] Objects such as these have been called $X$-shaped waves. An entire field of study and useful applications such as ultrasound scanning have resulted from the study of these $\mathrm{X}$-shaped waves. Localized X-shaped waves have been shown to be superluminal [?].

[31] R. Feynman, R. Leighton, and M. Sands, The Feynman Lectures on Physics - HTML Edition, The Feynman Lectures on Physics No. v. 2 (California Institute of Technology, 2013).

[32] G. D. Maccarrone and E. Recami, Lett. Nuovo Cim. 34, 251 (1982). 